\title{
MicroRNAs in model and complex organisms
}

\author{
Hikmet Budak ${ }^{1}$ • Baohong Zhang ${ }^{2}$
}

Published online: 20 February 2017

(C) Springer-Verlag Berlin Heidelberg 2017

\begin{abstract}
Non-coding RNAs such as microRNAs (miRNAs) are very tiny ribonucleotides having an essential role in gene regulation at both post-transcriptional and translational levels. They are very conserved and expressed in worms, flies, plants, and mammals in a sequence-specific manner. Furthermore, it is now possible to clone miRNAs using the new genome editing tool CRISPR/cas9, which shows benefit in control of untargeted effect. In this special issue, we tried to cover researches associated with functional roles of miRNAs accross model and complex organisms.
\end{abstract}

Keywords miRNAs · Noncoding RNAs · CRISPR/Cas · Plant and human genome

In 2000, when scientist presented the first draft of human genome, they were stunned by the fact that more than $97 \%$ of that has no revealed protein coding function. This part, referred as "junk DNA" was thought as evolutionary remnants and natural parasitic part of human genome which can reproduce and spread themselves across the genome (Lander et al., 2001). However, this term of "junk DNA" have revealed a giant stumble in following years and in fact, the "junk DNA"

This article forms part of a special issue of Functional \& Integrative Genomics entitled "miRNA in model and complex organisms" (Issue Editors: Hikmet Budak and Baohong Zhang)

Hikmet Budak

hikmet.budak@montana.edu

1 Cereal Genomics Lab, Department of Plant Sciences and Plant Pathology, Montana State University, Bozeman, MT, USA

2 Department of Biology, East Carolina University, Greenville, NC 27858, USA have come out as an big source of hidden treasures which scientist are still trying to understand. Apparently, the "junk DNA" has many important regulatory functions for maintenance of cellular life at the expressional, transcriptional and translational levels and the pieces of treasures are still in the process of discovery.

In recent years, a critical hidden molecule from the pool of junk DNA is discovered; microRNAs (miRNAs). miRNAs are an extensive class of small regulatory RNAs, which are the keystones controlling gene expression at the post transcriptional levels (Alptekin et al. 2016; Budak et al. 2015a; Budak and Akpinar 2015). These tiny ribo-regulators are widely existed in all eukaryotic organisms and they are involved in the regulation of many important pathways both in animals and plants (Alptekin et al. 2017; Budak et al. 2014; Eulalio et al. 2008; Zhang 2015; Zhang et al. 2007). miRNAbased gene expression regulation is critical in several diseases such as cancer, Alzheimer, Parkinson and they possess a potential of use as "biomarkers" for detection of such diseases and disease stages (Bekris and Leverenz 2015; Kosaka et al. 2010). In plants, they are contributed to stress tolerance together with environmental adaptation process and they have revealed a huge potential for crop improvement with the shed of several researches (Akpinar et al. 2015; Akpinar and Budak 2016; Budak et al. 2015b; Budak and Akpinar 2011). Considering the essential roles of them in many aspect of molecular life, miRNAs have attracted a considerable attention from scientific research community as well as industries in past 15 years (Lucas and Budak 2012; Kantar et al. 2012; Budak and Kantar 2015); however, many crucial points about these molecules still remain elusive.

Considering the importance of miRNAs, this special issue focuses on some exciting research questions associated with functional roles of miRNAs both in animals and plants with the contribution of 18 valuable papers (Alptekin et al. 2016; 
Alptekin and Budak 2016; Azevedo-Pouly et al. 2016; Baddela et al. 2016; Deniz and Erman, 2016; Esmaeili et al. 2016; Ferdous et al. 2016; Giusti et al. 2016; Gu et al. 2016; Gupta et al. 2017; Liu et al. 2016; Qiu et al. 2016; Reis et al. 2016; Soltani et al. 2016; Wang et al. 2017; You et al. 2017; Zhang et al. 2016; Zhao et al. 2016): How are the miRNAs involved in drought tolerance in plants? What is the role of miRNAs under biotic stress conditions? Are miRNAs crucial for cellular maintenance and survival in cancer tissues? What is the role of miRNAs in the developmental processes? In addition to research papers, three review paper are included in the special issue where the several controversial issues associated with miRNAs are discussed in a comprehensive and comparative way (Alptekin et al. 2016; Deniz and Erman 2016; Reis 2016). Overall, this issue gathers the papers from world-wide leading scientists of miRNA researches with the aim of providing up-to-date answers to miRNA-associated questions. Here, we highlight some of the particular subjects from this issue:

In spite the presence of several hypothesis about history of miRNAs and gene silencing at the post transcriptional levels, there are many points remain unexplained and not well-understood, particularly in plants (Voinnet 2009; Alptekin et al. 2017). In this special issue, Alptekin and Budak investigates the origin of miRNAs in bread wheat by performing an in silico identification and comparative analysis of miRNA repertoires of bread wheat and its diploid progenitors/relatives; their results provided important insight into the diversification and distribution of miRNA genes, which should contribute to the elucidation of miRNA evolution of Poaceae family (Alptekin and Budak 2016). Also, Reis reviews entangled history of animal and plant miRNAs in the consideration of current refinements and new hypothesis regarding to evolution of miRNAs (Reis 2016). This paper also provided detail information about miRNA and siRNA biogenesis and particular proteins involved in the biogenesis pathway. Also, You and colleagues focused on transcription start site of the Arabidopsis miRNAs which provide insight about miRNA biogenesis (You et al. 2017). Furthermore, Deniz and Erman described the long non-coding RNA molecules, their function and specific association between miRNAs (Deniz and Erman, 2016). These papers also provided a collection of studies which might be useful for gaining comprehensive information about miRNA biogenesis, miRNA evolution, and association of miRNAs with other sRNA molecules.

miRNAs are essential elements of stress adaptation and cell survival for plants under both biotic and abiotic stress condition (Budak et al. 2015b). In this special issue, Alptekin and colleagues reviewed the current progress about abiotic stress associated miRNAs and their targets as well the potential application of miRNA-based biotechnology on improvement of crop tolerance to abiotic stresses (Alptekin et al. 2016; Zhang 2015), particularly in wheat and barley (Alptekin et al. 2016). In addition, six research papers explore the miRNA response to abiotic stress as well as hormone treatment in several plant species (Esmaeili et al. 2016; Ferdous et al. 2016; Giusti et al. 2016; Liu et al. 2016; Gupta et al. 2017; Zhao et al. 2016) and detected miRNAs have shown that they play important roles in plant response to environmental adaptation. Liu and colleagues surveyed the miRNAs responsive to water-deficiency stress in four type of durum wheat (Liu et al. 2016). Ferdous and colleagues showed that overexpression of miR827 confers the drought tolerance in barley and it represented a potential for maintenance of drought tolerance in cereals (Ferdous et al. 2016). In addition to the cereals, drought and osmotic stress associated miRNAs are also characterized in peach and almond (Esmaeili et al. 2016; Giusti et al. 2016). Also, Gupta and colleagues were analyzed and identified miRNAs and tasiRNAs under phosphate deficient conditions (Gupta et al. 2017). Furthermore, the miRNAs from grapevine berries in response to ethylene hormone treatment were surveyed by Zhao and colleagues (Zhao et al. 2016). Overall, these studies highlighted many miRNA candidates which might contribute crop improvement.

miRNAs have also been detected as showing a differential expression pattern under several diseases in humans and possess a potential to be used as a biomarker. $\mathrm{Gu}$ and colleagues showed that miR-106b/25 presents a oncogene potential by utilization of myeloma cells and they were able to decrease the malignant progression of cancer by inhibiting this miRNA family (Gu et al. 2016). In another study, Azevedo-Pouly and colleagues demonstrated that miR-216 and miR-217 expression was reduced in transgenic mouse models of pancreatic adenocarcinoma. Knockout of miR-216/miR-217 host gene was lethal at embryonic level (Azevedo-Pouly et al. 2016). Additionally, Soltani and colleagues discussed the role of miRNAs in imatinib-resistant chronic myeloid leukemia and their results shed a light to new therapeutical approaches for this type of cancer which might also be helpful for inhibition of potential anticancer drug resistance (Soltani et al. 2016).

miRNAs might also contribute the regulation of developmental pathways both in animals and plants. Baddela and colleagues researched on the miRNAs which are effective on the development of ovarian follicular granulosa cells (GC) and their maturation which are particularly located on the chromosome 21 in cows and chromosome 20 in buffalos (Baddela et al. 2016). In another study, Qiu and colleagues provided evidence on infection of a novel rhizobium, Azorhizobium caulinodans ORS571 on wheat and the potential role of miRNAs during maturation of symbiosis process (Qiu et al. 2016). Zhang and colleagues also identified miRNAs from parasitic plant toot-knot nematode Meloidogyne incognita which contribute the understanding of host-plant interaction process and development (Zhang et al. 2016). In addition to these valuable researches, Wang and colleagues investigated the miRNAs which are involved 
in oil and oleic acid production during embryogenesis in Carya cathayensis (Wang et al. 2017). Thus, these miRNAs showed the importance of miRNAs in developmental process both at organismal and inter-organismal level.

In summary, this issue highlights the importance and essentialness of miRNAs for regulation of many important processes such disease resistance, stress tolerance and development. Described miRNAs in the shed of several valuable research papers presents a huge potential for the solution of today's critical problems like drug resistance or crop improvement. It is highly likely that future decades will show us the utilization of miRNAs in every aspects of science from medicine to agriculture. Given the need for a revised naming prescription, mis-identification and annotation of current miRNAs, CRISPR/Cas9 system in gene editing should be an innovative strategy in modulation of miRNA expression in model and complex organisms. Recently, Chen et al. (2016) employed CRISPY/Cas9 to knockout miR-126a gene, their results show the expression of miR-126 is significantly low and the knockout of miR-126a severely affected the formation of parachordal lymphangioblasts and thoracic duct in zebrafish embryos (Chen et al. 2016). Disruption of miR-137 via CRISPR/Cas9 inhibited the apoptosis in A2780 ovarian cells (Li et al. 2016). We believe CRISPR/Cas9 is becoming a powerful tool for studying the function of miRNAs as well improving traits for disease and pests in plants.

\section{References}

Akpinar BA, Budak H (2016) Dissecting miRNAs in wheat D genome progenitor, Aegilops tauschii. Front Plant Sci 7:1-17. doi:10.3389/ fpls.2016.00606

Akpinar BA, Kantar M, Budak H (2015) Root precursors of microRNAs in wild emmer and modern wheats show major differences in response to drought stress. Funct. Integr. Genomics. doi:10.1007/ s10142-015-0453-0

Alptekin B, Budak H (2016) Wheat miRNA ancestors: evident by transcriptome analysis of A, B, and D genome donors. Funct. Integr. Genomics. doi:10.1007/s10142-016-0487-y

Alptekin B, Langridge P, Budak H (2016) Abiotic stress miRNomes in the Triticeae. Funct. Integr. Genomics:1-26. doi:10.1007/s10142016-0525-9

Alptekin B, Akpinar BA, Budak H (2017) A comprehensive prescription for plant miRNA identification. Front Plant Sci 7:1-2058. doi:10. 3389/fpls.2016.02058

Azevedo-Pouly ACP, Sutaria DS, Jiang J, Elgamal OA, Amari F, Allard $\mathrm{D}$ et al (2016) miR-216 and miR-217 expression is reduced in transgenic mouse models of pancreatic adenocarcinoma, knockout of miR-216/miR-217 host gene is embryonic lethal. Funct. Integr. Genomics. doi:10.1007/s10142-016-0512-1

Baddela VS, Onteru SK, Singh D (2016) A syntenic locus on buffalo chromosome 20: novel genomic hotspot for miRNAs involved in follicular-luteal transition. Funct. Integr. Genomics. doi:10.1007/ s10142-016-0535-7

Bekris LM, Leverenz JB (2015) The biomarker and therapeutic potential of miRNA in Alzheimer's disease. Neurodegener Dis Manag 5:6174. doi: $10.2217 / \mathrm{nmt} .14 .52$
Budak H, Akpinar A (2011) Dehydration stress-responsive miRNA in Brachypodium distachyon: evident by genome-wide screening of microRNAs expression. Omi A J Integr Biol 15:791-799. doi:10. 1089/omi.2011.0073

Budak H, Akpinar BA (2015) Plant miRNAs: biogenesis, organization and origins. Funct. Integr. Genomics. doi:10.1007/s10142-0150451-2

Budak H, Kantar M (2015) Harnessing NGS and big data optimally: comparison of miRNA prediction from assembled versus nonassembled sequencing data - the case of the grass Aegilops tauschii complex genome. Omi. A J. Integr. Biol. 19:407-415. doi:10.1089/ omi.2015.0038

Budak H, Khan Z, and Kantar M (2014) History and current status of wheat miRNAs using next-generation sequencing and their roles in development and stress. Brief. Funct. Genomics, elu021-. doi:10. 1093/bfgp/elu021

Budak H, Bulut R, Kantar M, and Alptekin B (2015a). MicroRNA nomenclature and the need for a revised naming prescription. Brief. Funct. Genomics, elv026. doi:10.1093/bfop/elv026

Budak H, Kantar M, Bulut R, Akpinar BA (2015b) Stress responsive miRNAs and isomiRs in cereals. Plant Sci 235:1-13. doi:10.1016/ j.plantsci.2015.02.008

Chen J, Zhu RF, Li FF, Liang YL, Wang C, Qin YW, Huang S, Zhao XX, Jing Q (2016) MicroRNA-126a directs lymphangiogenesis through interacting with chemokine and Flt4 signaling in Zebrafish. Arterioscler Thromb Vasc Biol 36:2381-2393

Deniz E, Erman B (2016) Long noncoding RNA (lincRNA), a new paradigm in gene expression control. Funct. Integr. Genomics:1-9. doi: 10.1007/s10142-016-0524-x

Esmaeili F, Shiran B, Fallahi H, Mirakhorli N, Budak H, and MartínezGómez P (2016). In silico search and biological validation of microRNAs related to drought response in peach and almond. Funct. Integr. Genomics, 1-13. doi:10.1007/s10142-016-0488-x

Eulalio A, Huntzinger E, Izaurralde E (2008) Getting to the root of miRNA-mediated gene silencing. Cell 132:9-14. doi:10.1016/j. cell.2007.12.024

Ferdous J, Whitford R, Nguyen M, Brien C, Langridge P, Tricker PJ (2016) Drought-inducible expression of Hv-miR827 enhances drought tolerance in transgenic barley. Funct. Integr. Genomics. doi:10.1007/s10142-016-0526-8

Giusti L, Mica E, Bertolini E, De Leonardis AM, Faccioli P, Cattivelli L, et al. (2016). microRNAs differentially modulated in response to heat and drought stress in durum wheat cultivars with contrasting water use efficiency. Funct. Integr. Genomics 1-17. doi:10.1007/ s10142-016-0527-7

Gu C, Li T, Yin Z, Chen S, Fei J, Shen J, et al. (2016) Integrative analysis of signaling pathways and diseases associated with the miR-106b/25 cluster and their function study in berb erine-induced multiple myeloma cells. Funct. Integr. Genomics. doi:10.1007/s10142-0160519-7

Gupta S, Kumari M, Kumar H, Varadwaj PK (2017) Genome-wide analysis of miRNAs and Tasi-RNAs in Zea mays in response to phosphate deficiency. Funct. Integr. Genomics. doi:10.1007/s10142016-0538-4

Kantar M, Akpinar BA, Valárik M, Lucas SJ, Doležel J, Hernández P et al (2012) Subgenomic analysis of microRNAs in polyploid wheat. Funct. Integr. Genomics 12:465-479. doi:10.1007/s10142-0120285-0

Kosaka N, Iguchi H, Ochiya T (2010) Circulating microRNA in body fluid: a new potential biomarker for cancer diagnosis and prognosis. Cancer Sci 101:2087-2092. doi:10.1111/j.1349-7006. 2010.01650.x

Lander ES, Linton LM, Birren B, Nusbaum C, Zody MC, Baldwin J et al (2001) Initial sequencing and analysis of the human genome. Nature 409:860-921. doi:10.1038/35057062 
Li X, Chen W, Zeng W, Wan C, Duan S, and Jiang S 2016 microRNA137 promotes apoptosis in ovarian cancer cells via the regulation of XIAP. Br. J. Cancer, doi: 10.1038/bjc.2016.379

Liu H, Able AJ, Able JA (2016) Water-deficit stress-responsive microRNAs and their targets in four durum wheat genotypes. Funct. Integr. Genomics:1-15. doi:10.1007/s10142-016-0515-y

Lucas SJ, Budak H (2012) Sorting the wheat from the chaff: identifying miRNAs in genomic survey sequences of Triticum aestivum chromosome 1AL. PLoS One 7. doi:10.1371/journal.pone.0040859

Qiu L, Li Q, Zhang J, Chen Y, Lin X, Sun C et al (2016) Migration of endophytic diazotroph Azorhizobium caulinodans ORS571 inside wheat (Triticum aestivum L) and its effect on microRNAs. Funct. Integr. Genomics. doi:10.1007/s10142-016-0534-8

Reis RS (2016) The entangled history of animal and plant microRNAs. Funct. Integr. Genomics 1928:1-8. doi:10.1007/s10142-016-0513-0

Soltani I, Gharbi H, Hassine I. Ben, Bouguerra G, Douzi K, Teber M, et al. (2016). Regulatory network analysis of microRNAs and genes in imatinib-resistant chronic myeloid leukemia. Funct. Integr. Genomics 1. doi:10.1007/s10142-016-0520-1

Voinnet O (2009) Origin, biogenesis, and activity of plant microRNAs. Cell 136:669-687. doi:10.1016/j.cell.2009.01.046
Wang Z, Huang R, Sun Z, Zhang T, and Huang J (2017) Identification and profiling of conserved and novel microRNAs involved in oil and oleic acid production during embryogenesis in Carya cathayensis Sarg. Functional \& Integrative Genomics

You Q, Yan H, Liu Y, Yi X, Zhang K, Xu W, and Su Z (2017) A systemic identification approach for priTSS of Arabidopsis miRNAs from multidimensional omics data. Functional \& Integrative Genomics, under review

Zhang B (2015) MicroRNA: a new target for improving plant tolerance to abiotic stress. J Exp Bot 66:1749-1761. doi:10.1093/jxb/erv013

Zhang B, Wang Q, Pan X (2007) MicroRNAs and their regulatory roles in animals and plants. J Cell Physiol 210:279-289. doi:10.1002/jcp. 20869

Zhang Y, Wang Y, Xie F, Li C, Zhang B, Nichols RL et al (2016) Identification and characterization of microRNAs in the plant parasitic root-knot nematode Meloidogyne incognita using deep sequencing. Funct Integr Genomics:127-142. doi:10.1007/s10142015-0472-x

Zhao F, Wang C, Han J, Zhu X, Li X, Wang X et al (2016) Characterization of miRNAs responsive to exogenous ethylene in grapevine berries at whole genome level. Funct Integr Genomics. doi:10.1007/s10142-016-0514-z 